\title{
Efficacy and Safety Trends with Continuous, Long- Term Crisaborole Use in Patients Aged $\geq 2$ Years with Mild-to-Moderate Atopic Dermatitis
}

\author{
Bob Geng • Adelaide A. Hebert - Liza Takiya - Lauren Miller • \\ John L. Werth · Chuanbo Zang • Paul Sanders · Mark G. Lebwohl
}

Received: May 10, 2021 / Accepted: July 21, 2021

(C) The Author(s) 2021, corrected publication 2022

\begin{abstract}
Introduction: Atopic dermatitis (AD) is a chronic inflammatory skin disease often requiring long-term treatment. Crisaborole significantly improved global AD signs and symptoms in 28-day phase 3 studies of patients aged $\geq 2$ years with mild-to-moderate $\mathrm{AD}$ (Investigator's Static Global Assessment [ISGA] 2 or 3). A post hoc analysis of a long-term, openlabel extension study was conducted to assess
\end{abstract}

B. Geng

Division of Allergy, Immunology, and

Rheumatology, Department of Pediatrics, UC San

Diego School of Medicine, San Diego, CA, USA

A. A. Hebert

Department of Dermatology, UTHealth McGovern

School of Medicine, Houston, TX, USA

L. Takiya $(\bowtie) \cdot$ J. L. Werth · C. Zang

Pfizer Inc., Collegeville, PA, USA

e-mail: liza.takiya@pfizer.com

L. Miller

Dermatology Specialists, Gadsden, AL, USA

P. Sanders

Pfizer R\&D UK Ltd., Tadworth, Surrey, UK

M. G. Lebwohl

Department of Dermatology, Icahn School of

Medicine at Mount Sinai, New York, NY, USA efficacy and safety trends of crisaborole in patients stratified by the number of initial consecutive crisaborole treatment cycles, defined as the number of treatment cycles completed before achievement of ISGA 0 (clear)/1 (almost clear).

Methods: Patients completing phase 3 studies without drug-related safety issues that precluded further crisaborole treatment were analyzed. Patients with ISGA 0/1 at baseline (the end of a 28-day cycle) did not receive crisaborole for the next 28-day cycle (off-treatment), whereas patients with ISGA $\geq 2$ received crisaborole for the next 28-day cycle (on-treatment). Patients were stratified by number of initial consecutive crisaborole treatment cycles. Efficacy was assessed by achievement and maintenance of ISGA $0 / 1$, and safety was assessed by incidence of treatment-emergent adverse events (TEAEs) and treatment-related AEs (TRAEs).

Results: Overall, 418 patients were included in exclusive cohorts based on number of consecutive on-treatment cycles (1 on-treatment cycle, $n=133 ; \quad 2$ consecutive on-treatment cycles, $n=106 ; 3$ consecutive on-treatment cycles, $n=106 ; 4$ consecutive on-treatment cycles, $n=73$ ). After one to four initial consecutive ontreatment cycles, $77.6,76.3,59.4$, and $43.1 \%$ of patients, respectively, achieved ISGA 0/1. Of these patients, $49.5,37.8,44.4$, and $45.2 \%$, respectively, maintained ISGA $0 / 1$ at the end of a 28-day cycle off-treatment. Incidence of TRAEs was $4.5,4.7,3.8$, and $1.4 \%$ for patients 
receiving one to four consecutive on-treatment cycles, respectively. One patient discontinued because of AEs.

Conclusion: These results support the efficacious and safe continuous, long-term use of crisaborole for the management of mild-tomoderate AD.

Trial Registration: ClinicalTrials.gov: NCT021 18766, NCT02118792.

\section{PLAIN LANGUAGE SUMMARY}

Eczema is a skin disease that often requires longterm treatment. Crisaborole ointment improved mild-to-moderate eczema after 28 days of continuous use in two phase 3 clinical trials that included patients aged $\geq 2$ years. In the study reported here we tested whether eczema improved with continuous crisaborole use after one to four back-to-back treatment periods, or 28-112 days. Patients who completed either of the aforementioned phase 3 clinical trials were included in this study. Patients received crisaborole for the next 28-day period if they had eczema rashes. Patients with clear or almost clear skin did not receive crisaborole for 28 days. Our study included a total of 418 patients. After one to four treatment periods, $78,76,59$, and $43 \%$ of patients, respectively, had clear or almost clear skin. Of these patients, 50, 38, 44, and $45 \%$ still had clear or almost clear skin after stopping treatment for 28 days. Fewer than one in 20 patients had side effects related to crisaborole after one to four treatment periods. The most common side effect at the application site that was related to crisaborole was pain, particularly stinging and burning. Up to one in 50 patients had application site pain. One patient stopped taking crisaborole because of side effects. In conclusion, these results suggest that long-term crisaborole use is effective and safe.

Keywords: Atopic dermatitis; Crisaborole; Eczema; Maintenance therapy; Long-term

\section{Key Summary Points}

\section{Why carry out this study?}

Approval of crisaborole ointment, 2\%, for the treatment of patients with mild-tomoderate eczema was based on the results of two identically designed, vehiclecontrolled, 28-day studies of crisaborole in patients aged $\geq 2$ years with mild-tomoderate eczema.

Until now, the long-term efficacy and safety of crisaborole following continuous use had not been investigated.

The objective of this study was to evaluate the efficacy and safety of crisaborole in patients who received one to four consecutive crisaborole treatment cycles during a 48-week extension study.

\section{What was learned from the study?}

Many patients achieved clear or almost clear skin following one to four consecutive crisaborole treatment cycles (77.6, 76.3, 59.4, and 43.1\%, respectively), and treatment-related adverse events occurred in $<5 \%$ of patients across cohorts.

The results of this study support the efficacious and safe long-term, continuous use of crisaborole in patients who may require long treatment periods to manage the signs and symptoms of atopic dermatitis.

\section{INTRODUCTION}

Atopic dermatitis (AD) is a chronic inflammatory skin disease that occurs in as many as $15-30 \%$ of children and $2-10 \%$ of adults, with prevalence increasing worldwide [1-3]. Although the need for long-term treatment of $\mathrm{AD}$ is evident, the two most commonly recommended topical medications-topical corticos- 
teroids (TCS) and topical calcineurin inhibitors (TCI) - are recommended only for short-term, noncontinuous use because of safety concerns [3-6]. For example, long-term use of high-potency TCS can cause skin atrophy, striae, telangiectasia, perioral dermatitis, and numerous other adverse effects, and TCIs have a boxed warning of risk for malignancy [5-7]. As a result, there is a need for effective and safe long-term treatment options for patients with $\mathrm{AD}$.

Crisaborole ointment, $2 \%$, is a nonsteroidal phosphodiesterase 4 inhibitor for the treatment of mild-to-moderate AD. In two identically designed, 28-day, vehicle-controlled, phase 3 studies, crisaborole was well tolerated, and its use significantly improved global disease severity and all measured signs and symptoms of $\mathrm{AD}$ in patients aged $\geq 2$ years with mild-to-moderate $\mathrm{AD}$ [8]. The most common treatment-related adverse event (AE) was application site pain ( $4.4 \%$ crisaborole vs. $1.2 \%$ vehicle) [8]. In addition, an open-label, single-arm extension study was conducted to evaluate the safety of crisaborole. In this long-term study, treatmentrelated AEs that occurred over 48 weeks were similar to those reported in the pivotal studies, with treatment-related application site pain occurring in $2.3 \%$ of patients [9]. Crisaborole was more recently evaluated in a phase 4 study in infants (aged 3 months to $<2$ years) that demonstrated a similar safety profile and a rate of treatment-related application site pain of $3.6 \%[10]$.

To observe treatment effects of crisaborole with longer, continuous use than what was assessed in the 28-day phase 3 studies, we conducted a post hoc analysis of the long-term, open-label study in patients aged $\geq 2$ years to evaluate efficacy and safety trends during ontreatment and off-treatment cycles by examining those patients stratified by the number of initial consecutive treatment cycles.

\section{METHODS}

\section{Study Design and Patients}

The design of the extension study has been reported [9]. Briefly, this multicenter, single- arm, open-label, 48-week study (CORE 3) enrolled patients who completed a pivotal study (CrisADe CORE 1 [ClinicalTrials.gov: NCT02118766]; CrisADe CORE 2 [ClinicalTrials.gov: NCT02118792]) [8] without any drugrelated safety issues that precluded further treatment with crisaborole. Patients could have been off treatment between the pivotal studies and the long-term extension study for a maximum of 16 days. For the purposes of this analysis, patients were stratified by the number of initial consecutive on-treatment cycles required to achieve an Investigator's Static Global Assessment (ISGA) score of 0 (clear) or 1 (almost clear) (Fig. 1). Stratified groups were cohort 1 (those with 1 initial on-treatment cycle [i.e., 28-day initial on-treatment period]), cohort 2 (those with 2 initial consecutive on-treatment cycles [i.e., 56-day initial on-treatment period]), cohort 3 (those with 3 initial consecutive ontreatment cycles [i.e., 84-day initial on-treatment period]), and cohort 4 (those with 4 initial consecutive on-treatment cycles [i.e., 112-day initial on-treatment period]). Patients with no on-treatment cycles $(n=12)$ were not included in the analysis. In addition, patients with 5-12 initial consecutive on-treatment cycles $(n=87)$ were not included in the analysis because of limited sample size in each of the cohorts ( $n=25,22,16,4,3,2,4$, and 11, respectively).

The Quorum Review Institutional Review Board approved the protocol at each site. Written informed consent was obtained from patients or guardians. The study was conducted in accordance with the principles of Good Clinical Practice, the ethical principles originating in the Declaration of Helsinki, and all regional regulatory requirements.

\section{Treatment}

During participation in the pivotal studies, patients were randomly assigned to receive either crisaborole or vehicle. During the extension study, all patients with ISGA $\geq 2$ were treated with open-label crisaborole applied topically twice daily to all treatable AD-involved areas, excluding the scalp. Patients were instructed to apply crisaborole approximately 


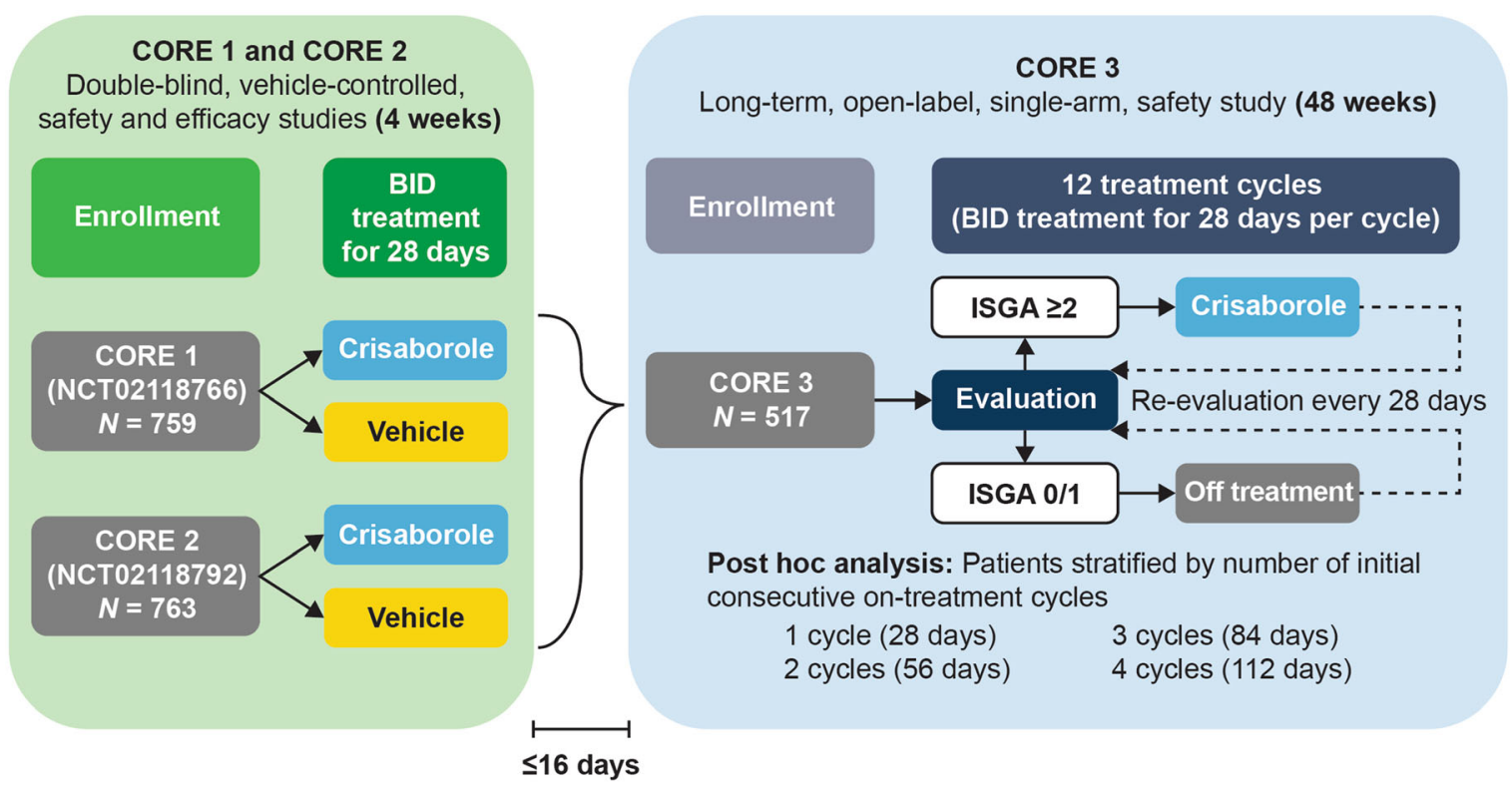

Fig. 1 Study design. BID twice daily, ISGA Investigator's Static Global Assessment

8-16 $\mathrm{h}$ after the morning dose and to avoid application of crisaborole to mucous membranes and eyes. On day 1 and at the end of each 28-day cycle over the 48-week study period, disease severity was evaluated using ISGA. If a patient's ISGA score was $\geq 2$, the patient received crisaborole twice daily for the next 28-day cycle (i.e., an "on-treatment period"); patients could have continued on crisaborole twice daily for additional 28-day cycles if the ISGA score was $\geq 2$ at the end of subsequent 28-day cycles. However, if the patient's ISGA score was 0 (clear) or 1 (almost clear), the patient did not receive crisaborole for the next 28-day cycle (i.e., an "off-treatment period") (Fig. 1). Both the on-treatment and off-treatment periods could last for multiple 28-day cycles, up to 48 weeks. Crisaborole treatment was discontinued if patients experienced no improvement in ISGA score after three consecutive on-treatment periods. Crisaborole was applied twice daily during on-treatment cycles regardless of the presence of active lesions and was not stopped when acute lesions resolved. Furthermore, application of crisaborole to treatable $\mathrm{AD}$ lesions identified after day 1 was permitted.

During off-treatment periods, patients could use bland (nonmedicated) emollients on all areas as needed. Bland emollients could be applied during on-treatment periods only on areas surrounding, but not on or overlapping, the treatable AD-involved areas. During ontreatment or off-treatment periods, use of rescue medications (low-to-mid potency TCSs or TCIs) was permitted and could be prescribed at the investigator's discretion if the patients were deemed to have intolerable AD. Crisaborole was discontinued during the use of rescue medications. Patients were permitted to use crisaborole after treatment with rescue medications if their ISGA score was $\geq 2$.

In total, patients might have received up to 12 cycles ( 28 days per cycle) of crisaborole. The study was considered completed when at least 100 patients finished approximately 1 year of follow-up.

\section{Endpoints}

Efficacy endpoints included the proportion of patients achieving ISGA $0 / 1$ at the end of their initial on-treatment period and, among those who had ISGA $0 / 1$ at the end of the initial ontreatment period, the following endpoints: the proportion of patients who maintained ISGA $0 / 1$ following 28 days off treatment, the 
proportion of patients who restarted treatment after their first off-treatment period, the number of off-treatment cycles before the restart of treatment, and the proportion of patients who regained ISGA $0 / 1$ at the end of the first retreatment cycle. Safety endpoints included the incidence of treatment-emergent adverse events (TEAEs) and treatment-related AEs (TRAEs) reported during the initial consecutive on-treatment period, including incidence rates of TEAEs per 100 patient-cycles.

\section{Statistical Analysis}

For this post hoc analysis, crude incidence rates of TEAEs per 100 patient-cycles and exact Poisson $95 \%$ confidence intervals were calculated. All other data were summarized descriptively.

\section{RESULTS}

\section{Patients}

A total of 418 patients received crisaborole for one to four consecutive initial on-treatment cycles and were included in this analysis. When stratified by number of initial consecutive treatment cycles, 133 patients each had one initial on-treatment cycle, 106 had two initial consecutive on-treatment cycles, 106 had three initial consecutive on-treatment cycles, and 73 had four initial consecutive on-treatment cycles. Study discontinuation rates during the initial on-treatment period were low and ranged from 2.7 to $5.7 \%$ across cohorts, with only one patient (in cohort 4) discontinuing because of an AE. Study discontinuation rates after the initial on-treatment period were markedly higher and ranged from 39.6 to $53.4 \%$ across cohorts (Fig. 2). The reasons for most of these discontinuations were "withdrawn consent" and "other," the latter of which comprised sponsor closure of the study (>95\%), investigator decision not related to safety, patient needing prohibited long-term medication, patient use of prohibited medication per protocol, and patient nonadherence to study visits. The number of study discontinuations because of AEs was low in all four cohorts during the ontreatment period $(<1.5 \%)$ and the off-treatment period $(<3 \%)$. Baseline characteristics across groups were similar except for the distribution of ISGA scores that demonstrated a numerically higher percentage of patients with moderate or severe disease (higher ISGA score) with the increasing number of initial consecutive on-treatment cycles (Table 1).

\section{Investigator's Static Global Assessment}

The proportion of patients who achieved clear or almost clear skin (ISGA 0/1) at the end of the initial on-treatment period ranged from $77.6 \%$ in those with one initial on-treatment cycle to $43.1 \%$ in those with four initial consecutive ontreatment cycles (Fig. 3a). Of these patients, ISGA $0 / 1$ was maintained in $49.5,37.8,44.4$, and $45.2 \%$ in cohorts 1 through 4 , respectively, at the end of a 28-day cycle off treatment (Fig. 3b).

Among those who achieved ISGA 0/1 at the end of their initial on-treatment period, the proportion of patients who reinitiated treatment at some point after an off-treatment cycle ranged from 77.4 to $87.3 \%$ among the four cohorts (Table 2). The mean number of cycles during which these patients were off treatment before restarting was approximately two, regardless of cohort (Table 2). On restarting treatment, the proportion of patients achieving ISGA $0 / 1$ by the end of one treatment cycle ranged from 37.5 to $53.3 \%$ among the cohorts, with higher rates in those who had one initial treatment cycle.

\section{Safety and Tolerability}

The incidence rate (per 100 patient-cycles) of TEAEs during the initial on-treatment period ranged from 12.9 to 23.4 across cohorts (Table 3). The most frequently reported TEAEs ( $\geq 5 \%$ in any group) during the initial ontreatment period were "viral upper respiratory tract infections," "cough," and "dermatitis atopic"; the incidence of each was $<7 \%$ in any group (Table 3 ). 


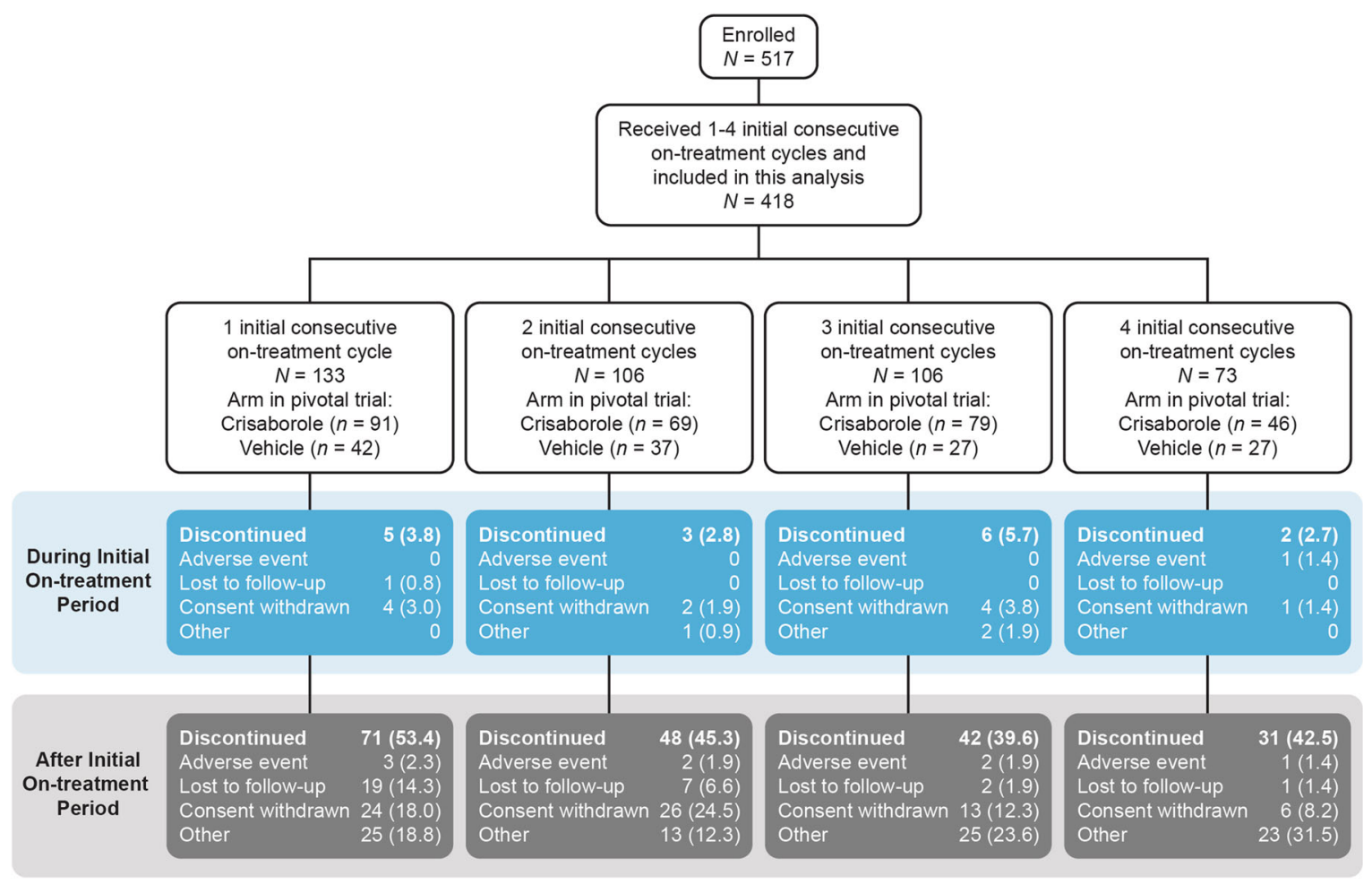

Fig. 2 Patient disposition. Other reasons for discontinuation included sponsor closure of the study, investigator decision not related to safety, long-term prohibited

The incidence rate (per 100 patient-cycles) of treatment-related AEs was low across all 4 cohorts and decreased with increasing number of initial consecutive on-treatment cycles (5.1 for cohort 1, 2.5 for cohort 2, 1.3 for cohort 3 , 0.3 for cohort 4 ) (Table 3 ). The most frequently reported treatment-related $\mathrm{AE}(\geq 2 \%$ in any group) during the initial on-treatment period was "dermatitis atopic," ranging from 0.9 to $3.8 \%$ among the four cohorts (Table 3). The incidence of individual treatment-related application site reactions (including "pain," "pruritus," and "paresthesia") during the initial on-treatment period was $<2 \%$ in all groups (Table 3).

\section{DISCUSSION}

In the primary phase 3 studies, patients aged $\geq 2$ years were treated continuously for 28 days, and approximately $50 \%$ of crisaborole- medication needed, use of prohibited medication per protocol, and nonadherence to study visits

treated patients were able to achieve ISGA 0/1 by day 28 [8]. In this post hoc analysis of the subsequent long-term study [9], it was possible to examine longer periods of continuous treatment with crisaborole. The results of our analysis suggest that, although not all patients achieved ISGA $0 / 1$ within the first month of treatment, longer-term (beyond 28 days) treatment with crisaborole was necessary in some patients to resolve the signs and symptoms of $\mathrm{AD}$, especially for patients with greater disease severity at baseline. Subsequently, after these longer continuous treatment periods, some patients maintained ISGA $0 / 1$ without treatment for 1 month, as seen by the 37.8 to $49.5 \%$ of patients who had clear or almost clear skin (ISGA 0/1) at the end of one off-treatment cycle. This short-term remission may be related to continuous use of therapy beyond the resolution of active lesions during the previous ontreatment cycles. However, most (77.4-87.3\%) of these patients had to restart treatment an 
Table 1 Demographics and baseline disease characteristics across groups

\begin{tabular}{|c|c|c|c|c|}
\hline & \multicolumn{4}{|c|}{ Initial on-treatment period } \\
\hline & $\begin{array}{l}\text { Cohort } 1 \\
(n=133)\end{array}$ & $\begin{array}{l}\text { Cohort } 2 \\
(n=106)\end{array}$ & $\begin{array}{l}\text { Cohort } 3 \\
(n=106)\end{array}$ & $\begin{array}{l}\text { Cohort } 4 \\
(n=73)\end{array}$ \\
\hline \multicolumn{5}{|c|}{ Baseline in CORE 1/CORE 2 studies } \\
\hline Age, mean (SD), years & $11.4(10.1)$ & $13.7(12.2)$ & $10.9(9.9)$ & $11.0(9.8)$ \\
\hline White, $n(\%)$ & $85(63.9)$ & $67(63.2)$ & $66(62.3)$ & $40(54.8)$ \\
\hline Female, $n(\%)$ & $78(58.7)$ & $62(58.5)$ & $58(54.7)$ & $45(61.6)$ \\
\hline BMI, mean (SD), $\mathrm{kg} / \mathrm{m}^{2}$ & $21.6(7.2)$ & $21.8(6.8)$ & $20.4(6.0)$ & $19.6(6.4)$ \\
\hline \%BSA, mean (SD) & $18.3(18.8)$ & $18.4(16.4)$ & $20.6(19.6)$ & $19.6(17.3)$ \\
\hline Prior use of TCSs, $n(\%)$ & $56(42.1)$ & $41(38.7)$ & $36(34.0)$ & $31(42.5)$ \\
\hline Prior use of TCIs, $n(\%)$ & $5(3.8)$ & $1(0.9)$ & $1(0.9)$ & $5(6.9)$ \\
\hline \multicolumn{5}{|l|}{ Baseline in CORE 3 study } \\
\hline \multicolumn{5}{|l|}{ ISGA scale, $n(\%)$} \\
\hline Clear $(0)$ & $7(5.3)$ & $6(5.7)$ & $3(2.8)$ & $1(1.4)$ \\
\hline Almost clear (1) & $18(13.5)$ & $10(9.4)$ & $4(3.8)$ & $1(1.4)$ \\
\hline Mild (2) & $61(45.9)$ & $47(44.3)$ & $46(43.4)$ & $21(28.8)$ \\
\hline Moderate (3) & $44(33.1)$ & $42(39.6)$ & $50(47.2)$ & $46(63.0)$ \\
\hline Severe (4) & $3(2.3)$ & $1(0.9)$ & $3(2.8)$ & $4(5.5)$ \\
\hline ISGA, mean (SD) & $2.1(0.9)$ & $2.2(0.9)$ & $2.4(0.7)$ & $2.7(0.7)$ \\
\hline
\end{tabular}

$\% B S A$ percentage of treatable body surface area, BMI body mass index, ISGA Investigator's Static Global Assessment, $S D$ standard deviation, TCI topical calcineurin inhibitor, TCS topical corticosteroid

average of 2 months ( 2 cycles) later. Substantial proportions of these patients were able to reach ISGA $0 / 1$ within 28 days ( 1 cycle) of restarting crisaborole, although this was more pronounced in the cohort who required only one initial cycle of treatment to achieve ISGA 0/1 (45.9\% of those patients had mild AD). A greater proportion of patients who received four initial consecutive treatment cycles had more severe disease than patients who received one initial treatment cycle; $68.5 \%$ of patients who received four initial consecutive treatment cycles had ISGA 3 (moderate) or 4 (severe), which could have accounted for the delayed response to crisaborole treatment. Still, the rates of achieving ISGA 0/1 within 28 days ( 1 cycle) showed that not as much time was required to reachieve ISGA $0 / 1$ for some patients who restarted therapy, even if they required two or more cycles to achieve ISGA 0/1 initially.

Crisaborole was well tolerated during the initial on-treatment period (i.e., 1-4 months), regardless of the number of initial consecutive on-treatment cycles. AE rates were consistent with those observed during the 28-day studies [8], and no new safety signals were observed. It is notable that $\mathrm{AE}$ rates were similar for patients who used crisaborole continuously for four cycles and for those who used crisaborole for one cycle, suggesting that crisaborole may be suitable therapy for AD even when used consistently for $>28$ days. The safety profile is particularly amenable for patients who might require longer-term continuous treatment to achieve adequate response and disease control. Use of crisaborole as maintenance or 


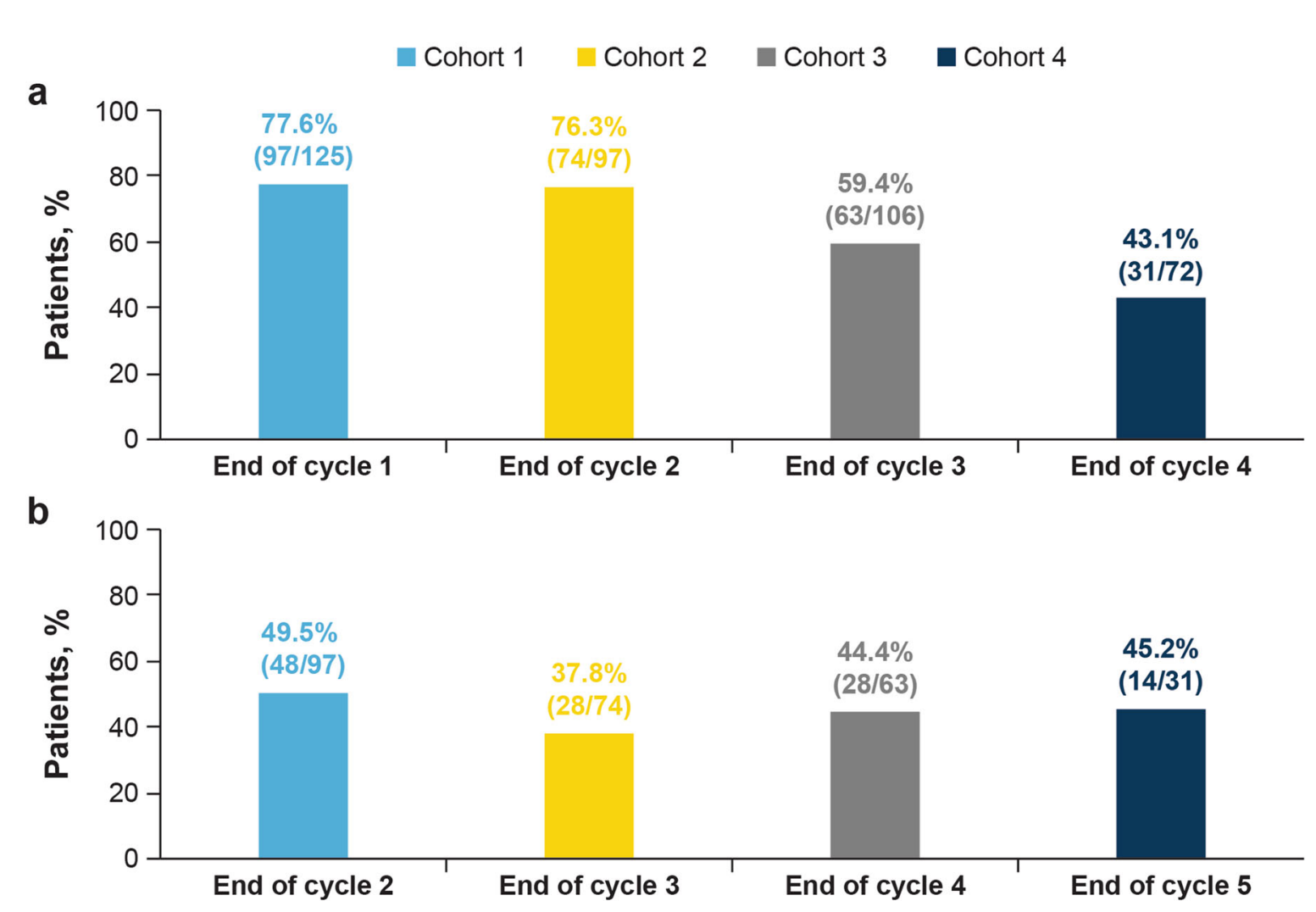

Fig. 3 Proportion of patients achieving ISGA $0 / 1$ at the end of their on-treatment period (a) and after 28 days off treatment (b) among those who previously achieved ISGA 0/1. ISGA Investigator's Static Global Assessment

Table 2 Treatment patterns following the initial on-treatment period

\begin{tabular}{lllll}
\hline Treatment patterns & Cohort 1 & Cohort 2 & Cohort 3 & Cohort 4 \\
\hline Restarted treatment, $n / N(\%)^{\mathrm{a}}$ & $77 / 97(79.4)$ & $60 / 74(81.1)$ & $55 / 63(87.3)$ & $24 / 31(77.4)$ \\
Consecutive cycles off treatment between & end of initial on-treatment period and restart of treatment & \\
$N$ & 97 & 74 & 63 & 31 \\
Mean (SD) & $2.8(2.8)$ & $2.2(2.5)$ & $2.3(2.0)$ & $2.7(2.3)$ \\
Median (range) & $1(0-11.0)$ & $1(0-10.0)$ & $1(1.0-9.0)$ & $1(1.0-8.0)$ \\
$\begin{array}{l}\text { Achieved ISGA 0/1 at end of first } \\
\text { re-treatment cycle, } n / N(\%)^{\mathrm{b}}\end{array}$ & $41 / 77(53.3)$ & $26 / 60(43.3)$ & $25 / 55(45.5)$ & $9 / 24(37.5)$ \\
\hline
\end{tabular}

$I S G A$ Investigator's Static Global Assessment, SD standard deviation

${ }^{a} n$ indicates the number of patients who restarted crisaborole treatment in each cohort; $N$ indicates the total number of patients who achieved ISGA $0 / 1$ in each cohort

${ }^{\mathrm{b}} n$ indicates the number of patients who achieved ISGA $0 / 1$ in each cohort following the first re-treatment cycle; $N$ indicates the total number of patients who restarted crisaborole treatment in each cohort 
Table 3 Adverse events during the initial on-treatment period

\begin{tabular}{|c|c|c|c|c|}
\hline & \multicolumn{4}{|c|}{ Initial on-treatment period } \\
\hline & $\begin{array}{l}\text { One cycle } \\
(n=133)\end{array}$ & $\begin{array}{l}\text { Two consecutive } \\
\text { cycles }(n=106)\end{array}$ & $\begin{array}{l}\text { Three consecutive } \\
\text { cycles }(n=106)\end{array}$ & $\begin{array}{l}\text { Four consecutive } \\
\text { cycles }(n=73)\end{array}$ \\
\hline Any TEAE, $n(\%)$ & $25(18.8)$ & $33(31.1)$ & $35(33.0)$ & $31(42.5)$ \\
\hline Patient-cycles & 107.0 & 175.7 & 272.0 & 228.8 \\
\hline Incidence rate $(95 \% \mathrm{CI})$ & $23.4(15.1-34.5)$ & $18.8(12.9-26.4)$ & $12.9(9.0-17.9)$ & $13.6(9.2-19.2)$ \\
\hline \multicolumn{5}{|c|}{ Most frequently reported ( $\geq 5 \%$ in any group) TEAEs, $n(\%)$} \\
\hline $\begin{array}{l}\text { Viral upper respiratory } \\
\text { tract infection }\end{array}$ & $1(0.8)$ & $3(2.8)$ & $6(5.7)$ & $1(1.4)$ \\
\hline Cough & $3(2.3)$ & $1(0.9)$ & $2(1.9)$ & $5(6.8)$ \\
\hline Dermatitis atopic & $5(3.8)$ & $5(4.7)$ & $8(7.5)$ & $5(6.8)$ \\
\hline Any TRAE, $n(\%)$ & $6(4.5)$ & $5(4.7)$ & $4(3.8)$ & $1(1.4)$ \\
\hline Patient-cycles & 118.4 & 201.9 & 318.9 & 292.6 \\
\hline Incidence rate $(95 \% \mathrm{CI})$ & $5.1(1.9-11.0)$ & $2.5(0.8-5.8)$ & $1.3(0.3-3.2)$ & $0.3(0.0-1.9)$ \\
\hline \multicolumn{5}{|c|}{ Most frequently reported ( $\geq 2 \%$ in any group) TRAEs, $n(\%)$} \\
\hline Dermatitis atopic & $2(1.5)$ & $4(3.8)$ & $1(0.9)$ & $1(1.4)$ \\
\hline \multicolumn{5}{|c|}{ Treatment-related application site reactions, $n(\%)$} \\
\hline $\begin{array}{l}\text { Application site } \\
\text { dermatitis }\end{array}$ & 0 & 0 & 0 & 0 \\
\hline Application site pain & $1(0.8)$ & $1(0.9)$ & $2(1.9)$ & 0 \\
\hline $\begin{array}{l}\text { Application site } \\
\text { paraesthesia }\end{array}$ & $1(0.8)$ & 0 & 0 & 0 \\
\hline Application site pruritus & 0 & 0 & $2(1.9)$ & 0 \\
\hline
\end{tabular}

Patient-cycles were calculated from baseline in CORE 3 to the day of first event; if no event occurred, the initial ontreatment period was used. Incidence rate is the number of patients with events per 100 patient-cycles

$C I$ confidence interval, TEAE treatment-emergent adverse event, TRAE treatment-related adverse event

prophylactic therapy for $\mathrm{AD}$ should be studied further, considering that long-term use of another AD treatment option (TCS) is associated with possible withdrawal symptoms after discontinuation $[7,11]$.

Given that TCS and TCI are associated with safety concerns that restrict long-term use [3-6], crisaborole may serve as a viable treatment option for patients who require long-term, continuous treatment to manage mild-to-moderate $\mathrm{AD}$. The observation that a substantial proportion of patients who restarted crisaborole treatment achieved ISGA 0/1 within 28 days suggested that crisaborole efficacy was regained despite the chronicity of AD. Moreover, crisaborole demonstrated a favorable safety profile for up to 112 days. Further study of the longterm efficacy and safety of continuous crisaborole use for the treatment of patients with mild-to-moderate $\mathrm{AD}$ is warranted.

CORE 3 was an open-label, single-arm study in which all patients received unblinded crisaborole treatment, which limits the ability to contextualize the data. In addition, the patients enrolled in the study had to have completed one of the pivotal studies without safety 
concerns, thereby introducing potential selection bias. Furthermore, patients in CORE 3 were previously enrolled in a parent study (CORE 1 or CORE 2) whereby the patients received crisaborole or vehicle for 28 days. However, supplemental analyses differentiating the results between patients who were randomly assigned to crisaborole versus placebo in the lead-in studies found no discernible difference between these groups (data not shown). Disease severity was assessed globally rather than for a particular lesion, as is customary with studies that use the ISGA scale to measure disease severity; therefore, new lesions that developed gradually could have affected the determination of disease severity over time. Although the study was not specifically designed to evaluate efficacy, the clinical signs of $\mathrm{AD}$ were assessed every 28 days to determine whether a patient entered an on-treatment or off-treatment period for the next 28-day cycle. As these assessments were performed only every 28 days, it was not possible to determine how patients responded at shorter intervals. Moreover, the distribution of crisaborole- and vehicle-treated skin sites was not recorded during the study, precluding any evaluation of crisaborole response across skin sites. Finally, the data were not controlled for baseline disease severity, making it difficult to separate the effect of $\mathrm{AD}$ severity from the effect of longer-term treatment with crisaborole.

\section{CONCLUSION}

Following long-term continuous crisaborole use, a substantial proportion of patients with mild-to-moderate AD experienced improvement in $\mathrm{AD}$ severity. The results of this analysis also support the longer term, continuous use of crisaborole (up to four 28-day cycles) given that some patients may require longer periods of treatment to effectively manage their $\mathrm{AD}$ symptoms. During these extended periods, crisaborole was well tolerated and no safety signals were observed, suggesting that the patients who required longer durations of treatment could use crisaborole safely. In addition, because patients might discontinue treatment after initially achieving ISGA 0/1, it may be possible for some patients to maintain ISGA $0 / 1$ for approximately 1-2 months (i.e., have 1 or 2 offtreatment cycles before restarting) and then regain ISGA $0 / 1$ after crisaborole treatment is reinitiated.

\section{ACKNOWLEDGEMENTS}

Funding. This study and the Journal's Rapid Service fee were funded by Pfizer Inc.

Authorship. All named authors meet the International Committee of Medical Journal Editors (ICMJE) criteria for authorship for this article, take responsibility for the integrity of the work as a whole, and have given their approval for this version to be published.

Author Contributions. BG, LT, LM, JLW, and MGL contributed to the conceptualization and design of the study; BG, LM, and CZ performed the data analysis; and all authors contributed to data interpretation. All authors drafted and critically reviewed the manuscript and approved the final version.

Medical Writing Assistance. Medical writing support under the guidance of the authors was provided by Stephanie O. Agbu, PhD, Robert J. Schoen, PharmD, and Jennifer C. Jaworski, MS, at ApotheCom, San Francisco, CA, USA, and was funded by Pfizer Inc., New York, NY, USA, in accordance with Good Publication Practice (GPP3) guidelines (Ann Intern Med. 2015;163:461-464).

Disclosures. Bob Geng has received honoraria as a consultant for Pfizer Inc., and as a speaker/consultant for Regeneron and Sanofi Genzyme. Adelaide A. Hebert is an employee of UTHealth McGovern Medical School, which receives research funds from Pfizer Inc., Anacor, Brikell, Cutanea, Dermira, GSK, and Novan; has received honoraria as a member of data safety monitoring boards for Bausch Health (Valeant), GSK, and Regeneron-Sanofi; and has received honoraria from Pfizer Inc., Biofrontera, Cutanea, Dermira, Galderma, Eli Lilly, Ortho 
Dermatologics, and Pierre Fabre. Liza Takiya, John L. Werth, and Chuanbo Zang are employees and stockholders of Pfizer Inc. Lauren Miller is a consultant for Pfizer Inc., AbbVie, Amgen, Castle Bioscience, Ortho Dermatologics, Novartis, Regeneron, and Sanofi Genzyme; and is a speaker for Pfizer Inc., Amgen, Eli Lilly, Novartis, Regeneron, and Sanofi Genzyme. Paul Sanders is an employee and stockholder of Pfizer R\&D UK Ltd. Mark G. Lebwohl is an employee of Mount Sinai, which receives research funds from AbbVie, Amgen, Arcutis, Boehringer Ingelheim, Dermavant, Eli Lilly, Incyte, Janssen Research \& Development, LEO Pharma, Ortho Dermatologics, Pfizer, and UCB; and is a consultant for Pfizer Inc., Aditum Bio, Allergan, Almirall, Arcutis, Avotres Therapeutics, BirchBioMed, BMD Skincare, Boehringer Ingelheim, Bristol Myers Squibb, Cara Therapeutics, Castle Biosciences, Corrona, Dermavant, Evelo, Facilitate International Dermatologic Education, Foundation for Research and Education in Dermatology, Inozyme Pharma, LEO Pharma, Meiji Seika Pharma, Menlo, Mitsubishi, Neuroderm, Promius/Dr. Reddy's Laboratories, Serono, Theravance, and Verrica.

Compliance with Ethics Guidelines. Quorum Review Institutional Review Board approved the protocol at each site. Written informed consent was obtained from patients or guardians. The study was conducted in accordance with the principles of Good Clinical Practice, the ethical principles originating in the Declaration of Helsinki, and all regional regulatory requirements.

Data Availability. The datasets generated during and/or analyzed during the current study are not publicly available. Upon request, and subject to certain criteria, conditions and exceptions (see https://www.pfizer.com/ science/clinical-trials/trial-data-and-results for more information), Pfizer will provide access to individual de-identified participant data from Pfizer-sponsored global interventional clinical studies conducted for medicines, vaccines, and medical devices (1) for indications that have been approved in the USA and/or EU or (2) in programs that have been terminated (i.e., development for all indications has been discontinued). Pfizer will also consider requests for the protocol, data dictionary, and statistical analysis plan. Data may be requested from Pfizer trials 24 months after study completion. The deidentified participant data will be made available to researchers whose proposals meet the research criteria and other conditions, and for which an exception does not apply, via a secure portal. To gain access, data requestors must enter into a data access agreement with Pfizer.

Prior Presentation. This analysis was previously presented in part at the 2020 Virtual Annual Meeting of the American Academy of Allergy, Asthma, \& Immunology, 13-16 March 2020; the 33rd Annual Eastern Allergy Conference, 16-18 August 2020, Palm Beach, FL, USA; Maui Derm Virtual Congress, 24-27 June 2020; and the Revolutionizing Atopic Dermatitis 2020 Virtual Symposium, 5 April 2020.

Open Access. This article is licensed under a Creative Commons Attribution-NonCommercial 4.0 International License, which permits any non-commercial use, sharing, adaptation, distribution and reproduction in any medium or format, as long as you give appropriate credit to the original author(s) and the source, provide a link to the Creative Commons licence, and indicate if changes were made. The images or other third party material in this article are included in the article's Creative Commons licence, unless indicated otherwise in a credit line to the material. If material is not included in the article's Creative Commons licence and your intended use is not permitted by statutory regulation or exceeds the permitted use, you will need to obtain permission directly from the copyright holder. To view a copy of this licence, visit http://creativecommons.org/licenses/by$\mathrm{nc} / 4.0 /$.

\section{REFERENCES}

1. Bieber T. Atopic dermatitis. $\mathrm{N}$ Engl J Med. 2008;358(14):1483-94. 
2. Bieber T. Atopic dermatitis. Ann Dermatol. 2010;22(2):125-37.

3. Eichenfield LF, Tom WL, Berger TG, et al. Guidelines of care for the management of atopic dermatitis: section 2. Management and treatment of atopic dermatitis with topical therapies. J Am Acad Dermatol. 2014;71(1):116-32.

4. Schneider L, Tilles S, Lio P, et al. Atopic dermatitis: a practice parameter update 2012. J Allergy Clin Immunol. 2013;131(2):295-9 (e1-27).

5. Protopic [prescribing information]. Ballerup: LEO Pharma Inc.; 2019. https://www.leo-pharma.us/ Files/Billeder/US\%20Website\%20Product\%20PIs/ Protopic\%20Ointment\%20-\%20USPI\%20and\%20 Med\%20Guide\%20-\%20Feb\%202019\%20FINAL. pdf. Accessed 12 July 2021.

6. Elidel [prescribing information]. Bridgewater: Bausch Health; 2020. https://www.bauschhealth. com/Portals/25/Pdf/PI/Elidel-PI.pdf. Accessed 12 July 2021.

7. Hengge UR, Ruzicka T, Schwartz RA, Cork MJ. Adverse effects of topical glucocorticosteroids. J Am Acad Dermatol. 2006;54(1):1-15 (quiz 6-8).
8. Paller AS, Tom WL, Lebwohl MG, et al. Efficacy and safety of crisaborole ointment, a novel, nonsteroidal phosphodiesterase 4 (PDE4) inhibitor for the topical treatment of atopic dermatitis (AD) in children and adults. J Am Acad Dermatol. 2016;75(3):494-503.e6.

9. Eichenfield LF, Call RS, Forsha DW, et al. Long-term safety of crisaborole ointment $2 \%$ in children and adults with mild to moderate atopic dermatitis. J Am Acad Dermatol. 2017;77(4):641-9.e5.

10. Schlessinger J, Shepard JS, Gower R, et al. Safety, effectiveness, and pharmacokinetics of crisaborole in infants aged 3 to $<24$ months with mild-tomoderate atopic dermatitis: a phase IV open-label study (CrisADe CARE 1). Am J Clin Dermatol. 2020;21(2):275-84.

11. Sheary B. Steroid withdrawal effects following longterm topical corticosteroid use. Dermatitis. 2018;29(4):213-8. 